\title{
Phase Portraits of the Autonomous Duffing Single-Degree-of-Freedom Oscillator with Coulomb Dry Friction
}

\author{
Nikola Jakšić \\ Kolektor Etra, Šlandrova Ulica 10, 1231 Ljubljana-Črnuče, Slovenia \\ Correspondence should be addressed to Nikola Jakšić; nikola.jaksic@gmail.com
}

Received 13 May 2014; Accepted 18 August 2014; Published 14 September 2014

Academic Editor: Lars Hakansson

Copyright (C) 2014 Nikola Jakšić. This is an open access article distributed under the Creative Commons Attribution License, which permits unrestricted use, distribution, and reproduction in any medium, provided the original work is properly cited.

The paper presents phase portraits of the autonomous Duffing single-degree-of-freedom system with Coulomb dry friction in its $\delta-\gamma-\varepsilon$ parameter space. The considered nonlinearities of the cubic stiffness $(\varepsilon)$ and Coulomb dry friction $(\gamma)$ are widely used throughout the literature. It has been shown that there can be more than one sticking region in the phase plane. It has also been shown that an equilibrium point occurs at the critical combinations of values of the parameters $\gamma$ and $\varepsilon$ which gives rise to zero eigenvalue of the linearised system. The unstable limit cycle may appear in the case of negative viscous damping $(\delta) ; \delta<0$.

\section{Introduction}

It is beneficial, if not essential, to know the system behaviour prior to using it in any other application or analysis. Phase portraits of the autonomous Duffing single-degreeof-freedom oscillator with Coulomb dry friction are studied here in a complete parameter space.

In order to understand the phase portraits of the nonlinear system in the next section, it is necessary to review the phase portraits of the linear system at different parameter values.

Without loss of generality, the autonomous linear singledegree-of-freedom (SDOF) system's equation of motion can be written as follows:

$$
\ddot{x}+a \dot{x}+b x=0,
$$

where $a$ represents viscous damping in the system and $b$ represents system's stiffness. By defining the discriminant $D=$ $a^{2}-4 b$ the system eigenvalues $\lambda$ are as follows: $\lambda_{1}=(-a+$ $\sqrt{D}) / 2$ and $\lambda_{2}=(-a-\sqrt{D}) / 2$. If $D>0$ then $\lambda_{1}, \lambda_{2} \in \mathbb{R}$ and the response is $x(t)=A_{1} e^{\lambda_{1} t}+A_{2} e^{\lambda_{2} t}$. If $D=0$ then $\lambda_{1}=\lambda_{2}=\lambda \in \mathbb{R}$ and the response is $x(t)=\left(A_{1}+A_{2} t\right) e^{\lambda t}$. Finally if $D<0$ then the eigenvalues are complex conjugated, $\lambda_{1,2}=R \pm i I \in \mathbb{C}$, and the response is $x(t)=e^{R t}\left(A_{1} \cos (I t)+\right.$ $\left.A_{2} \sin (I t)\right) . A_{1}$ and $A_{2}$ are constants dependent on the initial conditions.
The equation of motion (1) can be represented as system of first-order differential equations:

$$
\dot{\mathbf{r}}=\mathbf{A r},
$$

where $\mathbf{r}=\{x, y\}$ and $y=\dot{x}$ and finally $\dot{\mathbf{r}}$ is the phase velocity. Hence,

$$
\mathbf{A}=\left[\begin{array}{cc}
0 & 1 \\
-b & -a
\end{array}\right]
$$

There are only two possibilities regarding the number of the equilibrium points [1]. Either there is only one equilibrium point at the origin of the phase plane or there is infinity number of equilibrium points situated at zero velocity abscissa of the phase plane. Table 1 presents all possibilities of the phase portraits of the linear system.

Some aspects of the viscous and frictional damping acting together are presented in [2] on the linear system with the Coulomb dry friction.

Negative viscous damping is normally associated with self-exciting systems. They are most commonly found in solid-fluid interactions with the fluid induced vibrations [3, $4]$, but certainly not exclusively $[5,6]$. There are two main reasons for using the self-excited model: either the excitation itself is too difficult to model or only the consequences of the energy-input mechanism are important for the problem under consideration. 
Both mechanisms of energy dissipation have been used in many studies concerning the forced system, for example, [7-9]. It was shown that separating the viscous damping and frictional damping from the system response is possible, but difficult $[10,11]$. Hence, the interactions between both mechanisms of energy dissipation should be well understood.

The mixed model (power law damping) has also received some attention $[12,13]$.

\section{Nonlinear System}

Two most commonly used nonlinearities, the cubic stiffness and the Coulomb dry friction, are added to the linear system, thus adding two terms to (1):

$$
\ddot{x}+2 \delta \omega \dot{x}+\omega^{2} x+\varepsilon x^{3}+\gamma \operatorname{sign}(\dot{x})=0,
$$

where $\delta$ stands for the viscous damping ratio $(2 \delta \omega$ is equivalent to $a$ ), $\omega$ for the undamped eigenfrequency of the equivalent linear system ( $\omega^{2}$ is equivalent to $\left.b\right), \varepsilon$ for the coefficient of nonlinear stiffness, and $\gamma$ for the equivalent friction force.

The Duffing oscillator has been widely studied in the literature; a lot of analyses of the Duffing system behaviour are gathered, ordered, and presented in [14].

Let us suppose that $\omega>0$ and $\gamma>0$ throughout the text. The use of Coulomb model of dry friction divides the phase plane into two half-planes: the positive half-plane for the positive velocities and the negative half-plane for the negative velocities. The nonsaddle equilibrium points of the SDOF system are shifted to the left from their previous position on the positive half-plane and toward the right on the negative half-plane and vice versa for the saddle points. Between these pairs of equilibrium points the sticking region is formed. Any trajectory in the phase plane that collides with the sticking region stays there forever. Coulomb dry friction model does not directly change the nature of the equilibrium points, as viscous damping does. It only changes their position [15]. The nature of some types of the equilibrium points is directly changed by the viscous damping parameter $\delta$ (or $a$ for the equivalent linear system); see Table 1 , column $b>0$. Hence, the equations of motion are

Positive half-plane $(y>0)$

$$
\begin{aligned}
& \dot{x}=y \\
& \dot{y}=-2 \delta \omega y-\omega^{2} x-\varepsilon x^{3}-\gamma
\end{aligned}
$$

Negative half-plane $(y<0)$

\begin{tabular}{|c|c|c|c|c|}
\hline & & $b<0$ & $b=0$ & $b>0$ \\
\hline$a<0$ & $\begin{array}{l}D>0 \\
D=0 \\
D<0\end{array}$ & Saddle & $\begin{array}{l}\text { Inf. num. of } \\
\text { equilibrium } \\
\text { points }\end{array}$ & $\begin{array}{c}\text { Unstable node } \\
\text { Unstable inflected node } \\
\text { Unstable focus }\end{array}$ \\
\hline \multicolumn{2}{|c|}{$a=0$} & Saddle & $\begin{array}{l}\text { Inf. num. of } \\
\text { equilibrium } \\
\text { points }\end{array}$ & Centre \\
\hline$a>0$ & $\begin{array}{l}D>0 \\
D=0 \\
D<0\end{array}$ & Saddle & $\begin{array}{l}\text { Inf. num. of } \\
\text { equilibrium } \\
\text { points }\end{array}$ & $\begin{array}{l}\text { Stable node } \\
\text { Sable inflected node } \\
\text { Stable focus }\end{array}$ \\
\hline
\end{tabular}

$$
\begin{aligned}
& \dot{x}=y \\
& \dot{y}=-2 \delta \omega y-\omega^{2} x-\varepsilon x^{3}+\gamma .
\end{aligned}
$$

TABLE 1: Equilibrium points of the linear system.

\section{Equilibrium Points}

The equilibrium points are obtained at the zero phase velocity:

$$
\begin{aligned}
& \text { Positive half-plane }(y>0) \\
& \dot{x}=0 \Longrightarrow y_{e}^{+}=0 \\
& \dot{y}=0 \Longrightarrow \omega^{2} x_{e}^{+}+\varepsilon\left(x_{e}^{+}\right)^{3}+\gamma=0 \\
& \text { Negative half-plane }(y<0) \\
& \dot{x}=0 \Longrightarrow y_{e}^{-}=0 \\
& \dot{y}=0 \Longrightarrow \omega^{2} x_{e}^{-}+\varepsilon\left(x_{e}^{-}\right)^{3}-\gamma=0,
\end{aligned}
$$

where superscript + stands for the positive phase half-plane and superscript - for the negative one. Index $e$ stands for the coordinates of the equilibrium points. Equation (6) gives rise to at most three equilibrium points at each half-plane. The nature of the equilibrium points can be deduced from linearized system, (7), linearized in the proximity of each of the equilibrium points, in the case of its nonzero eigenvalues. Consider the following:

$$
\mathbf{A}=\left[\begin{array}{cc}
0 & 1 \\
-\omega^{2}-3 \varepsilon\left(x_{e}^{ \pm}\right)^{2} & -2 \delta \omega
\end{array}\right]=\left[\begin{array}{cc}
0 & 1 \\
-B\left(x_{e}^{ \pm}\right) & -A
\end{array}\right]
$$

It is obvious that coefficients $B$ and $A$ from (7) directly correspond to the linear system coefficients $b$ and $a$, respectively; see (3) and Table 1.

The discriminant $\mathscr{D},(8)$, is defined first, when solving the cubic equation (6). The value of the discriminant $\mathscr{D},(8)$, determines the number of equilibrium points, for example, number of real solutions of cubic equation (6). Consider the following:

$$
\mathscr{D}=q^{2}+p^{3}=\frac{1}{\varepsilon^{2}}\left(\frac{\gamma^{2}}{4}+\frac{\omega^{6}}{27 \varepsilon}\right),
$$




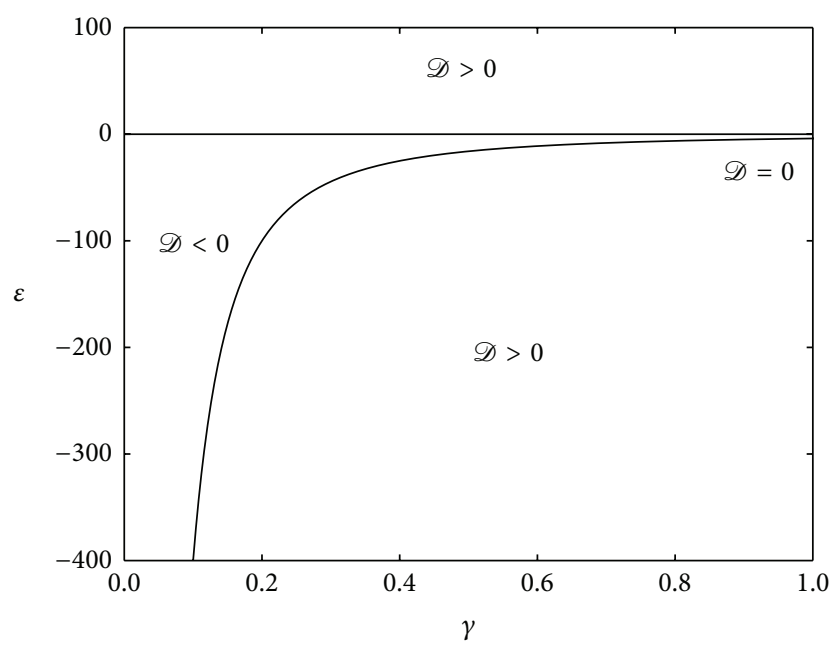

Figure 1: Discriminant $\mathscr{D}$ value in the $\gamma-\varepsilon$ parameter plane. Actual parameters' values: $\omega=\sqrt{3}$.

where

Positive half-plane $(y>0)$

$$
\begin{aligned}
& p^{+}=p=\frac{\omega^{2}}{3 \varepsilon} \\
& q^{+}=\frac{\gamma}{2 \varepsilon}
\end{aligned}
$$

Negative half-plane $(y<0)$

$$
\begin{aligned}
& p^{-}=p=\frac{\omega^{2}}{3 \varepsilon} \\
& q^{-}=-\frac{\gamma}{2 \varepsilon} .
\end{aligned}
$$

If $\mathscr{D}>0$ there is only one equilibrium point on each halfplane; on the other hand if $\mathscr{D}<0$ there are three equilibrium points on each half-plane. In the case of $\mathscr{D}=0$ there are two equilibrium points on each half-plane. Two of the equilibrium points, which exist at $\mathscr{D}<0$, collide and form an equilibrium point which cannot be analysed by the linearised system. The $\gamma-\varepsilon$ parameter plane is divided into subregions according to the discriminant's $\mathscr{D}$ sign; see Figure 1 . The zero discriminant $\mathscr{D}$ surface in the $\gamma-\omega$ - $\varepsilon$ parameter space is presented in Figure 2. The value of $\mathscr{D}$ as function of $\varepsilon$ at the given value of $\gamma$ is presented in Figure 3. The stability of the equilibrium points and their location as a function of the parameter $\varepsilon$ is presented in Figure 4. If $\delta<0$ then there are no stable equilibrium points.

3.1. Case A. One has $\varepsilon \geq 0$. In this case $\mathscr{D}>0$ holds and (6) has only one real solution for each half-plane. Consider the following:

$$
\begin{aligned}
& x_{A}^{+}=\sqrt[3]{-q^{+}+\sqrt{\mathscr{D}}}+\sqrt[3]{-q^{+}-\sqrt{\mathscr{D}}}<0 \\
& x_{A}^{-}=\sqrt[3]{-q^{-}+\sqrt{\mathscr{D}}}+\sqrt[3]{-q^{-}-\sqrt{\mathscr{D}}}>0 .
\end{aligned}
$$

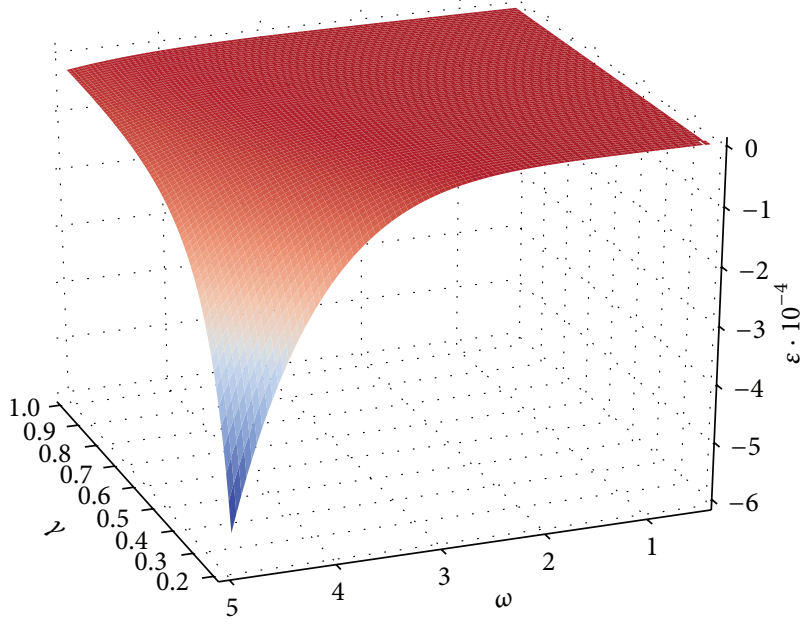

Figure 2: Zero discriminant $\mathscr{D}$ surface in the $\gamma-\omega-\varepsilon$ parameter space.

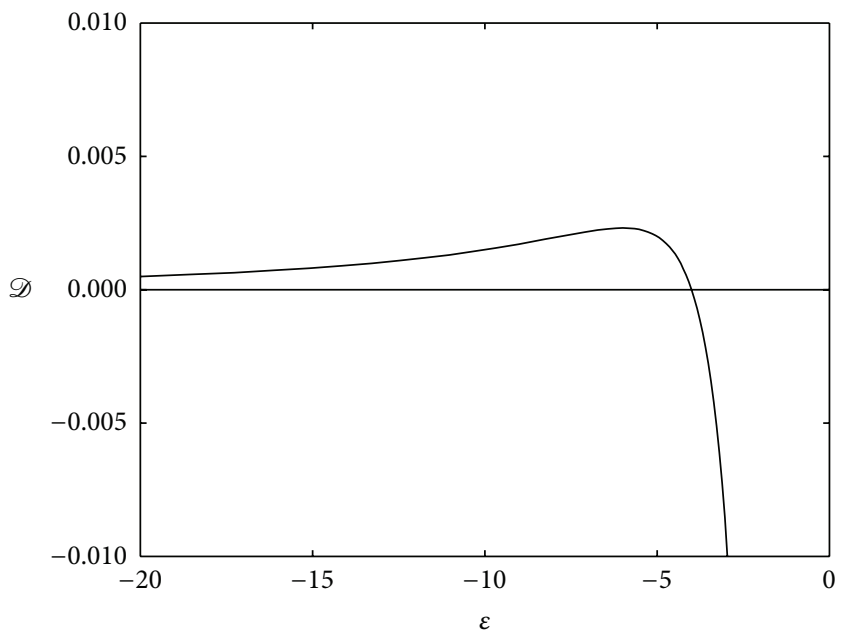

FIGURE 3: Discriminant $\mathscr{D}$ value as function of $\varepsilon$. Actual parameters' values: $\omega=\sqrt{3} ; \gamma=1$.

The constant $B\left(x_{A}^{ \pm} \equiv x_{e}^{ \pm}\right)$can be evaluated from (7) and that gives $B\left(x_{A}^{ \pm}\right)>0$, which defines both equilibrium points as centres if there is no viscous damping. In the presence of the viscous damping the nature of the equilibrium points changes as described by the column $b>0$ in Table 1 . Hence, the stability of the equilibrium points is governed by the viscous damping ratio $\delta$.

3.2. Case B. One has $\varepsilon<0$. There are three subcases depending on the discriminant's $\mathscr{D}$ sign. As fast as $\varepsilon$ parameter becomes negative, two unstable equilibrium points appear from $+\infty$, one for each half-plane, and other two from $-\infty$, again one for each half-plane, subcase B1. The absolute value of the $x_{e}$ for the four equilibrium points grows smaller with every smaller value of the parameter $\varepsilon$ as presented in Figure 4. At certain value of the parameter $\varepsilon$ two of the saddle equilibrium points collide with the stable equilibrium points 


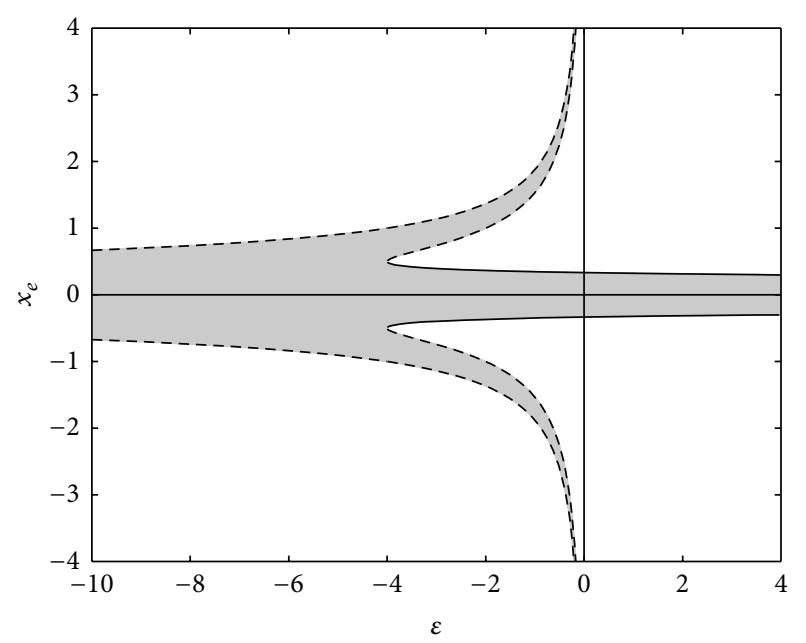

FIGURE 4: Location and stability of the equilibrium points as function of $\varepsilon$. Stable (-) if $\delta \geq 0$ and unstable if $\delta<0$ and unstable (- - ) equilibrium points. The sticking region is presented by the shaded region. Actual parameters' values: $\omega=\sqrt{3} ; \gamma=1$.

(at $\delta>0$ ), which exist also when $\varepsilon>0$, subcase B2. At this point only two unstable equilibrium points remain, subcase B3.

3.2.1. Subcase B1. One has $\mathscr{D}<0$. Equation (6) has three real solutions for each half-plane; see Figure 4. By defining

$$
\begin{gathered}
r=-\sqrt{|p|}=-\frac{\omega}{\sqrt{3|\varepsilon|}}<0 \\
\cos \varphi^{ \pm}=\frac{q^{ \pm}}{r^{3}}=\mp \frac{\gamma}{2 \omega^{3}} \sqrt{27|\varepsilon|}
\end{gathered}
$$

the abscises of the equilibrium points can be computed as follows:

$$
\text { Positive half-plane }(y>0)
$$

$$
\begin{aligned}
& x_{B 1_{1}}^{+}=-2 r \cos \frac{\varphi^{+}}{3}>0 \\
& x_{B 1_{2}}^{+}=-2 r \cos \left(\frac{\varphi^{+}-4 \pi}{3}\right)<0 \\
& x_{B 1_{3}}^{+}=-2 r \cos \left(\frac{\varphi^{+}-2 \pi}{3}\right)<0
\end{aligned}
$$

Negative half-plane $(y<0)$

$$
\begin{aligned}
& x_{B 1_{1}}^{-}=-2 r \cos \frac{\varphi^{-}}{3}>0 \\
& x_{B 1_{2}}^{-}=-2 r \cos \left(\frac{\varphi^{-}-4 \pi}{3}\right)<0 \\
& x_{B 1_{3}}^{-}=-2 r \cos \left(\frac{\varphi^{-}-2 \pi}{3}\right)>0 .
\end{aligned}
$$

Constants' $B\left(x_{B 1_{i}}^{ \pm} \equiv x_{e}^{ \pm}\right) ; i=1,2,3$ signs are estimated with the help of (6) and (7) as follows:

Positive half-plane $(y>0)$

$$
\begin{aligned}
& B\left(x_{B 1_{1}}^{+}\right)<0 \\
& B\left(x_{B 1_{2}}^{+}\right)<0 \\
& B\left(x_{B 1_{3}}^{+}\right)>0
\end{aligned}
$$

Negative half-plane $(y<0)$

$$
\begin{aligned}
& B\left(x_{B 1_{1}}^{-}\right)<0 \\
& B\left(x_{B 1_{2}}^{-}\right)<0 \\
& B\left(x_{B 1_{3}}^{-}\right)>0 .
\end{aligned}
$$

The first two equilibrium points on both phase half-planes are saddles. The last equilibrium point on both phase half-planes is centre if there is no viscous damping. In the presence of the viscous damping the nature of these equilibrium points changes as described by the column $b>0$ in Table 1 .

3.2.2. Subcase B2. One has $\mathscr{D}=0$. Equation (6) has three real solutions for each half-plane, but two of them are the same; see Figure 4. Consider the following:

Positive half-plane $(y>0)$

$$
\begin{aligned}
& x_{B 2_{1}}^{+}=2 \sqrt[3]{-q^{+}}=\sqrt[3]{-\frac{4 \gamma}{\varepsilon}}>0 \\
& x_{B 2_{2}}^{+}=x_{B 2_{3}}^{+}=\sqrt[3]{q^{+}}=\sqrt[3]{\frac{\gamma}{2 \varepsilon}}<0
\end{aligned}
$$

Negative half-plane $(y<0)$

$$
\begin{aligned}
& x_{B 2_{1}}^{-}=2 \sqrt[3]{-q^{-}}=\sqrt[3]{\frac{4 \gamma}{\varepsilon}}<0 \\
& x_{B 2_{2}}^{-}=x_{B 2_{3}}^{-}=\sqrt[3]{q^{-}}=\sqrt[3]{-\frac{\gamma}{2 \varepsilon}}>0 .
\end{aligned}
$$

Constants' $B\left(x_{B 2_{i}}^{ \pm} \equiv x_{e}^{ \pm}\right) ; i=1,2,3$ signs are estimated with the help of (7) as follows:

$$
\begin{gathered}
B\left(x_{B 2_{1}}^{ \pm}\right)=\omega^{2}+3 \varepsilon\left(x_{B 2_{1}}^{ \pm}\right)^{2}=-3 \omega^{2}<0 \\
B\left(x_{B 2_{2}}^{ \pm}\right)=B\left(x_{B 2_{3}}^{ \pm}\right)=\omega^{2}+3 \varepsilon\left(x_{B 2_{2,3}}^{ \pm}\right)^{2}=0 .
\end{gathered}
$$

It is clear from (15) that the first equilibrium point, $x_{B 2_{1}}^{ \pm}$, is saddle. Other two solutions give a single equilibrium point of higher order, which cannot be properly described by linearised system due to zero eigenvalue [16]. However, the centre manifold theorem $[17,18]$ allows us to assess the stability of such points. According to the centre manifold theorem the system has one centre manifold and one stable manifold at this point. Hence, the equilibrium points are not unstable. 
3.2.3. Subcase B3. One has $\mathscr{D}>0$. There is only one real solution of (6) for each half-plane in this case; see Figure 4. Consider the following:

$$
\begin{aligned}
& x_{B 3}^{+}=\sqrt[3]{-q^{+}+\sqrt{\mathscr{D}}}+\sqrt[3]{-q^{+}-\sqrt{\mathscr{D}}}>0 \\
& x_{B 3}^{-}=\sqrt[3]{-q^{-}+\sqrt{\mathscr{D}}}+\sqrt[3]{-q^{-}-\sqrt{\mathscr{D}}}<0 .
\end{aligned}
$$

The constant $B\left(x_{B 3}^{ \pm} \equiv x_{e}^{ \pm}\right)$can be evaluated from (7) and that gives $B\left(x_{B 3}^{ \pm}\right)<0$, which defines both equilibrium points as saddles.

\section{Sticking Regions}

The smooth transition of the trajectory from one half-plane to the other one is achieved when $\dot{y}^{+} \dot{y}^{-}>0$ and sticking appears when $\dot{y}^{+} \dot{y}^{-}<0$ [8]. Hence the sticking regions appear in between two equivalent equilibrium points each from different phase half-plane. The sticking region as function of parameter $\varepsilon$ is presented in Figure 4 as the shaded region.

\section{Phase Portraits and Basins of Attraction}

The phase portraits are important in understanding the global dynamics of the system, especially in the vicinity of the equilibrium points. Equilibrium points marked with the red circles belong to the positive half-plane and the ones marked with the blue squares to the negative half-plane of the phase plane. The basin of attraction is traced by numerical integration of the system response from the initial conditions. If the trajectory ends up in the attracting set the initial conditions are marked as a point in the phase plane. If the trajectory exhibits unstable motion the point is left unmarked. In the case where the whole phase plane is the basin of attraction no initial point is marked.

5.1. Case $A$. One has $\varepsilon \geq 0$. There are two equilibrium points, see Figure 4, which type is governed by the value of parameter $\delta$. Hence, four main subcases can be recognized here, defined by the value of the parameter $\delta$. When parameter $\delta$ reaches value of $\delta_{c r}^{+}=1.0$ the nature of the equilibrium points is changed from stable node to stable focus or vice versa. On the contrary, when parameter $\delta$ reaches value of $\delta_{c r}^{-} \approx-1.0885$ the limit cycle shrinks to the equilibrium points and ceased to exist. It is replaced by two heteroclinic orbits.

5.1.1. Subcase A1. One has $\delta>\delta_{c r}^{+}>0$. The phase portrait is presented in Figure 5. The equilibrium points are attractorsstable nodes and the sticking region is the attracting set. The basin of attraction is whole phase plane.

5.1.2. Subcase A2. One has $\delta_{c r}^{+}>\delta \geq 0$. The phase portrait is presented in Figure 6. The equilibrium points are attractorsstable focuses (centres if $\delta=0$ ) and the sticking region is the attracting set. The basin of attraction is whole phase plane again.

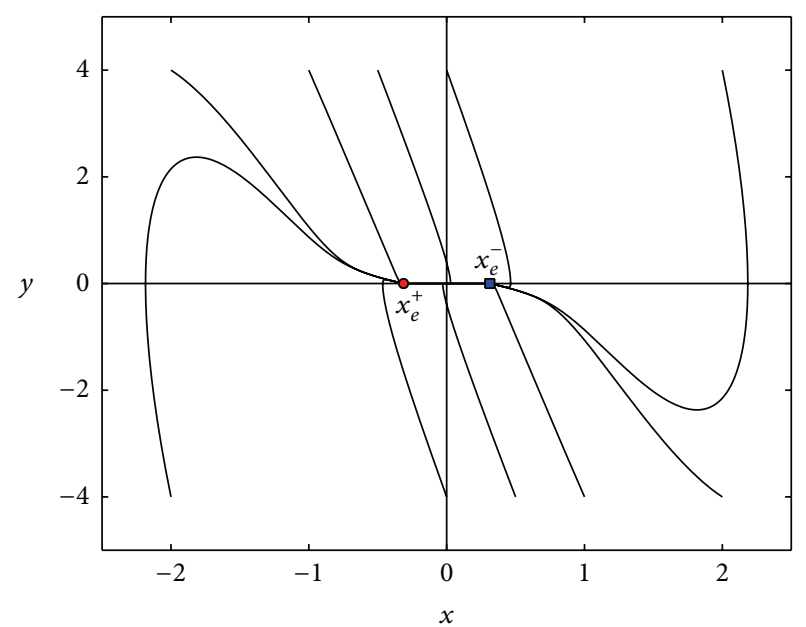

FIGURE 5: The phase portrait of case A1. Actual parameters' values: $\omega=\sqrt{3} ; \gamma=1 ; \delta=2$.

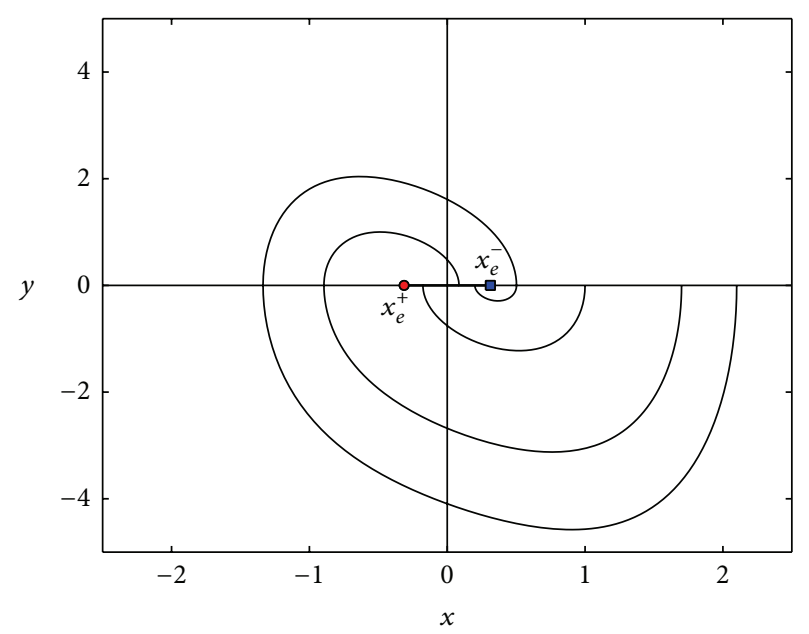

FIGURE 6: The phase portrait of case A2. Actual parameters' values: $\omega=\sqrt{3} ; \gamma=1 ; \delta=0.2$.

5.1.3. Subcase A3. One has $\delta_{c r}^{-}<\delta<0$. The phase portrait is presented in Figure 7. The equilibrium points are attractorsunstable focuses and the sticking region is the attracting set. The trajectory that repeats itself where repellency of the equilibrium points is balanced by the attraction of the sticking region can be found. The dry friction energy dissipation mechanism attracts the trajectories closer to the sticking region whereas the negative viscous damping mechanism repels them at the larger distances. Thus the unstable limit cycle forms where these two influences are in balance. The phenomenology is mathematically described in [2] for a simpler system. The amplitude of the limit cycle $x_{c}$ as a function of $\delta$ is presented in Figure 8. The basin of attraction lies within the limit cycle. The trajectories that start inside the limit cycle end up in the sticking region. The trajectories that start outside the limit cycle end up in $\pm \infty$. Hence, the limit cycle divides the phase plane into region of stable and unstable motion; see Figure 7. 


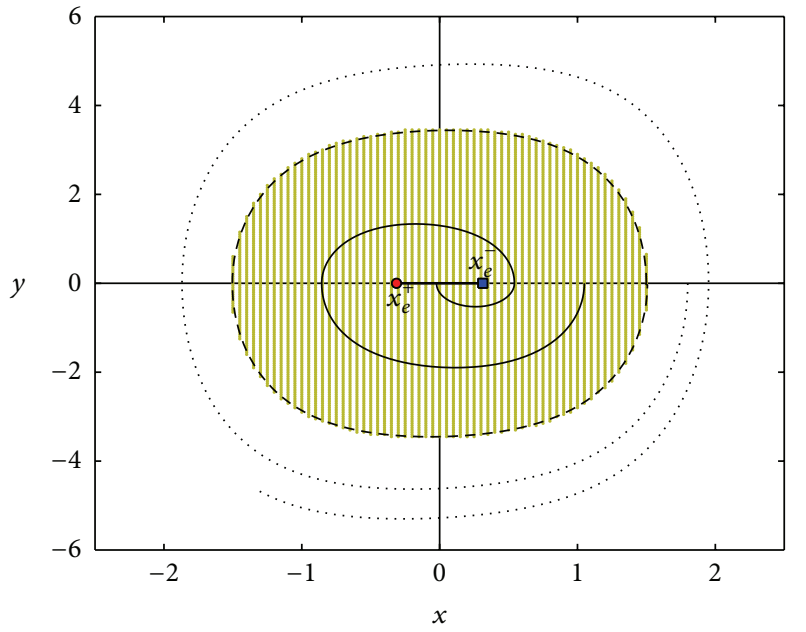

Figure 7: The phase portrait of case A3. - stable trajectory, ... unstable trajectory, - - - unstable limit cycle and yellow dots present the basin of attraction. Actual parameters' values: $\omega=\sqrt{3} ; \gamma=1$; $\delta=-0.2$

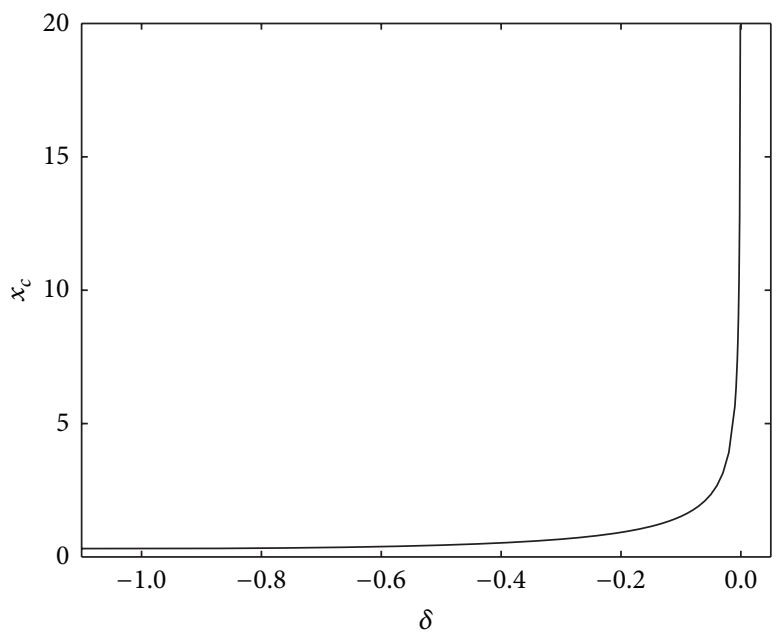

FIGURE 8: The amplitude $x_{c}$ of the limit cycle. Actual parameters' values: $\omega=\sqrt{3} ; \gamma=1$.

5.1.4. Subcase A4. One has $\delta<\delta_{c r}^{-}<0$. The phase portrait is presented in Figure 9. The equilibrium points are attractorsunstable nodes and the sticking region is the attracting set. Limit cycle from case A3 is shirked towards equilibrium points when $\delta$ approaches $\delta_{c r}^{-}$and vanishes when $\delta \leq \delta_{c r}^{-}$. The heteroclinic orbits divide the phase plane into regions of the stable motion and one of the unstable motion in this case, Figure 9. The phenomenology is mathematically described in [2] for a simpler system.

5.2. Case $B$. One has $\varepsilon<0$. There are three subcases, which depend upon the value of the discriminant $\mathscr{D}$. The value of the viscous damping $\delta$ has the largest impact in subcase B1 where $\mathscr{D}<0$ and no impact at all in subcase B3 where $\mathscr{D}>0$ and all equilibrium points are saddles.

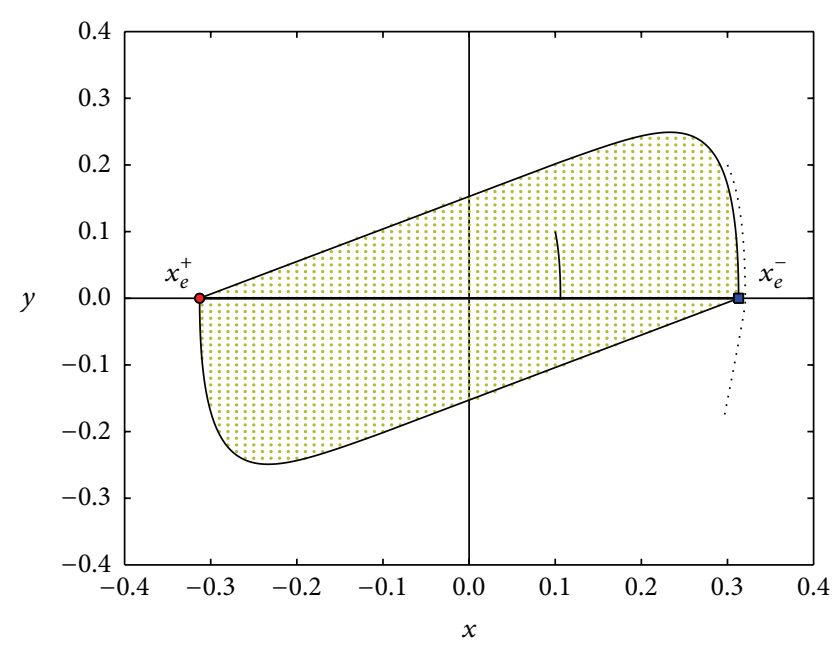

Figure 9: The phase portrait of case A4. - stable trajectory, $\cdots$ unstable trajectory, thick - heteroclinic orbits and yellow dots present the basin of attraction. Actual parameters' values: $\omega=\sqrt{3}$; $\gamma=1 ; \delta=-2$.

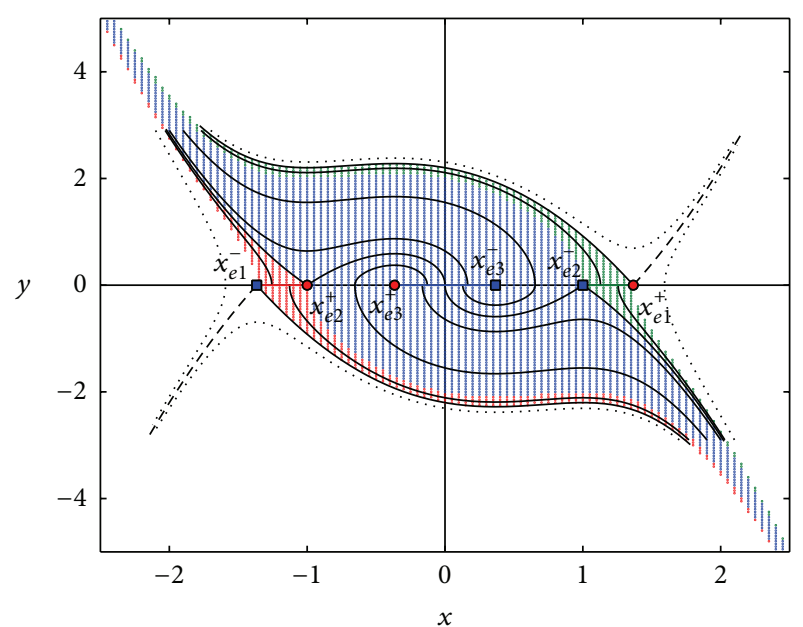

FIgUre 10: The phase portrait of case $\mathrm{B} 1$ at $\delta=0$. Thick - stable separatrix, thick - - unstable separatrix, - stable trajectory, $\cdots$ unstable trajectory and coloured dots present the basin of attraction of different attracting sets. Actual parameters' values: $\omega=\sqrt{3} ; \gamma=1$; $\varepsilon=-2 ; \delta=0$.

5.2.1. Subcase B1. One has $\mathscr{D}<0$. The phase portraits for values of $\delta>\delta_{c r_{A}} \approx-0.30405$ are qualitatively similar. The phase portrait for the value of $\delta=0$ is presented in Figure 10 and of $\delta=-0.25$ in Figure 11. The trajectory coming out from saddle points $x_{e 2}^{+}$and $x_{e 2}^{-}$ends up in the sticking region between equilibrium points $x_{e 3}^{+}$and $x_{e 3}^{-}$(blue region).

The first qualitative change happens at $\delta=\delta_{c r_{A}} \approx$ -0.30405 , Figure 12, where trajectory coming out from the saddle point $x_{e 2}^{+}$ends up in the saddle point $x_{e 2}^{-}$and thus becomes the heteroclinic orbit. The same goes for the trajectory from $x_{e 2}^{-}$to $x_{e 2}^{+}$.

When the value of $\delta$ is lessened further on, Figure 13, the trajectory coming out from saddle point $x_{e 2}^{+}$ends up in the 


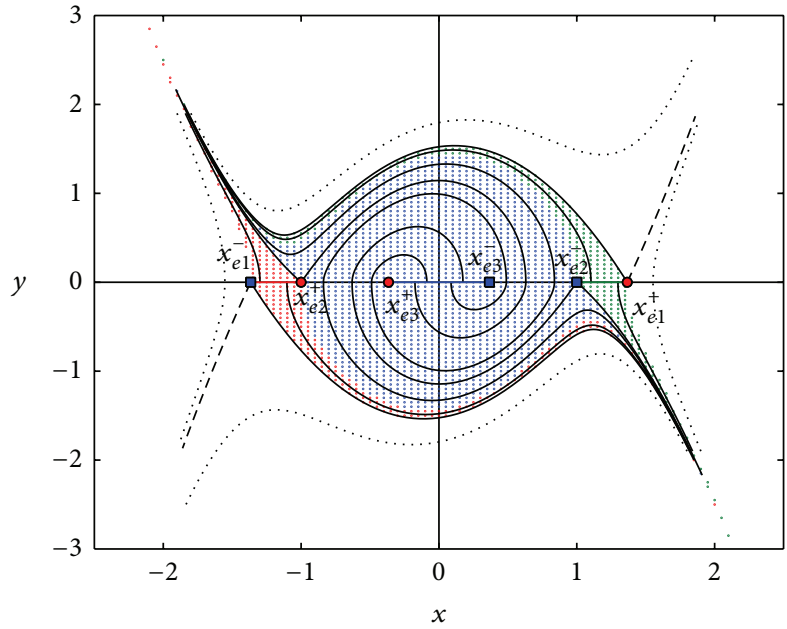

Figure 11: The phase portrait of case B1 at $\delta=-0.25$. Thick - stable separatrix, thick - - unstable separatrix, - stable trajectory, $\cdots$ unstable trajectory and coloured dots present the basin of attraction of different attracting sets. Actual parameters' values: $\omega=\sqrt{3} ; \gamma=1$; $\varepsilon=-2 ; \delta=-0.25$.

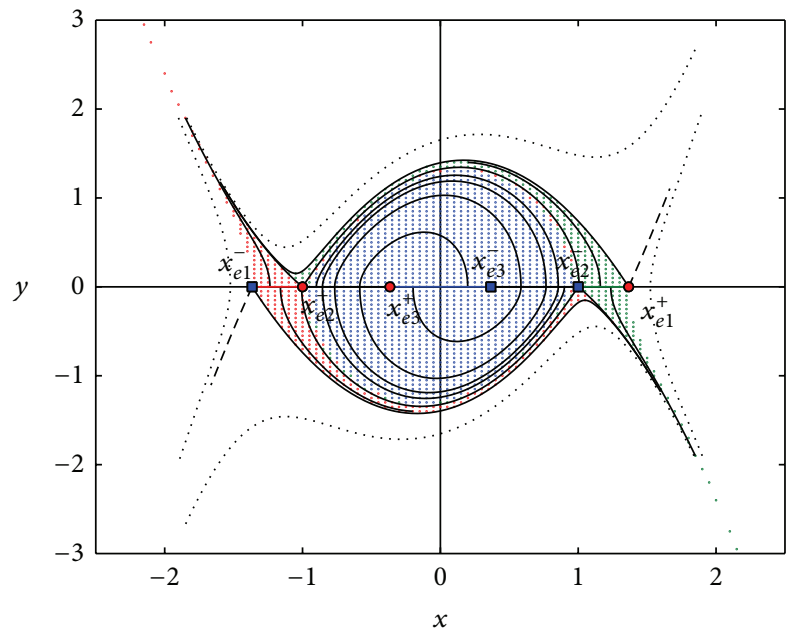

FIgURE 12: The phase portrait of case B1 at $\delta=-0.30405$. Thick stable separatrix, thick - - - unstable separatrix, - stable trajectory, $\cdots$ unstable trajectory and coloured dots present the basin of attraction of different attracting sets. Actual parameters' values: $\omega=$ $\sqrt{3} ; \gamma=1 ; \varepsilon=-2 ; \delta=-0.30405 \approx \delta_{c r_{A}}$.

sticking region between equilibrium points $x_{e 2}^{-}$and $x_{e 1}^{+}$(green region). And the trajectory coming out from saddle points $x_{e 2}^{-}$ends up in the sticking region between equilibrium points $x_{e 1}^{-}$and $x_{e 2}^{+}$(red region). The unstable limit cycle is formed between equilibrium points $x_{e 2}^{+}$and $x_{e 2}^{-}$.

Next qualitative change of the phase portrait happens at the value of $\delta=\delta_{c r_{B}} \approx-0.317$, Figure 14. Here the trajectory leaving the saddle point $x_{e 2}^{+}$ends up in the saddle point $x_{e 2}^{-}$ and thus becomes heteroclinic orbit. The same is true for the trajectory between $x_{e 2}^{-}$and $x_{e 2}^{+}$. The unstable limit cycle is still

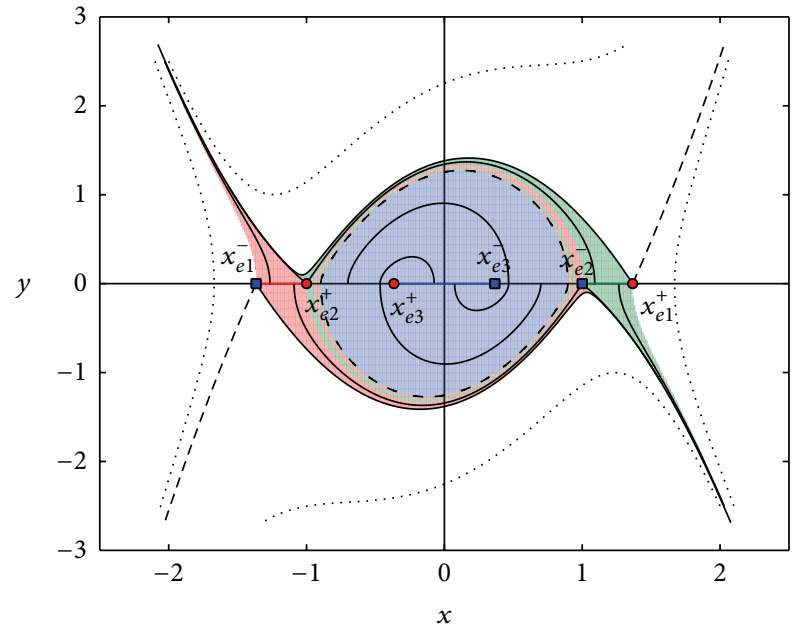

Figure 13: The phase portrait of case B1 at $\delta=-0.31$. Thick stable separatrix, thick - - - unstable separatrix, thick - $\cdots$ unstable limit cycle, - stable trajectory, $\cdots$ unstable trajectory and coloured dots present the basin of attraction of different attracting sets. Actual parameters' values: $\omega=\sqrt{3} ; \gamma=1 ; \varepsilon=-2 ; \delta=-0.31$.

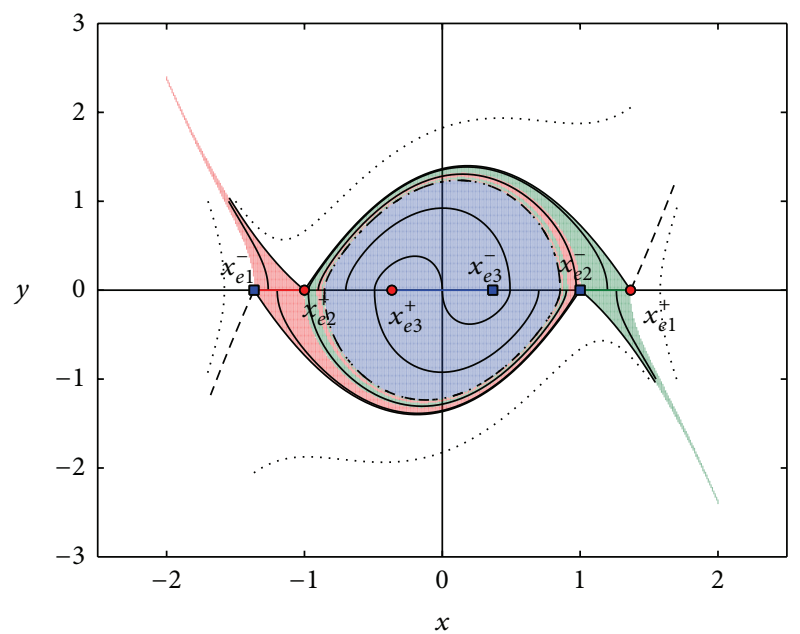

FIGURE 14: The phase portrait of case B1 at $\delta=-0.316975$. Thick stable separatrix, thick - - - unstable separatrix, thick - - unstable limit cycle, - stable trajectory, $\cdots$ unstable trajectory and coloured dots present the basin of attraction of different attracting sets. Actual parameters' values: $\omega=\sqrt{3} ; \gamma=1 ; \varepsilon=-2 ; \delta=-0.316975 \approx \delta_{c r_{B}}$.

present. It shrinks in comparison with the limit cycle at larger $\delta$.

Beyond $\delta=\delta_{c r_{B}} \approx-0.317$ the basins of attraction split among themselves, Figure 15. The unstable limit cycle shrinks further on.

The limit cycle shrinks toward equilibrium points and ceased to exist at $\delta=\delta_{c r_{C}} \approx-0.8660$, Figure 16. There are two heteroclinic orbits linking $x_{e 3}^{+}$and $x_{e 3}^{-}$instead of the limit cycle. The phase portraits with even smaller $\delta$ do not change qualitatively. 


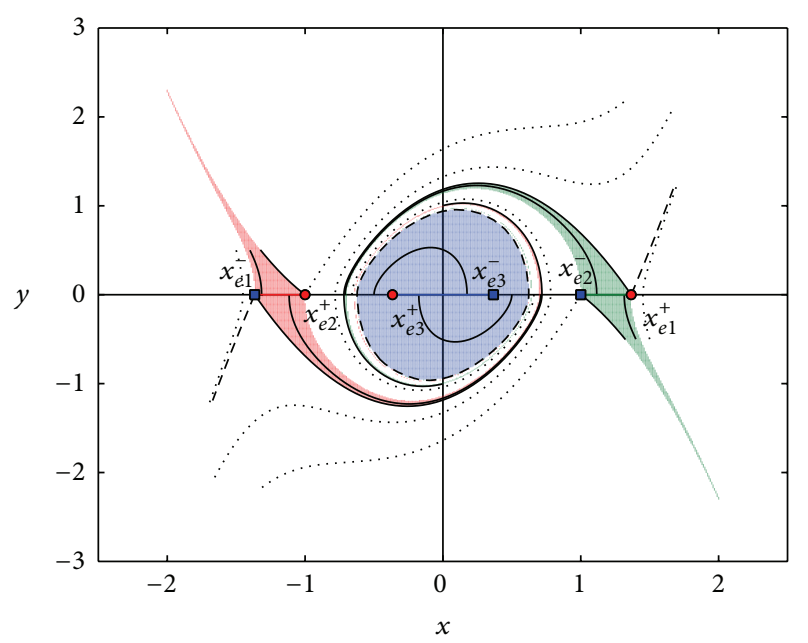

Figure 15: The phase portrait of case B1 at $\delta=-0.4$. Thick - stable separatrix, thick - - - unstable separatrix, thick - - unstable limit cycle, - stable trajectory, $\cdots$ unstable trajectory and coloured dots present the basin of attraction of different attracting sets. Actual parameters' values: $\omega=\sqrt{3} ; \gamma=1 ; \varepsilon=-2 ; \delta=-0.4$.

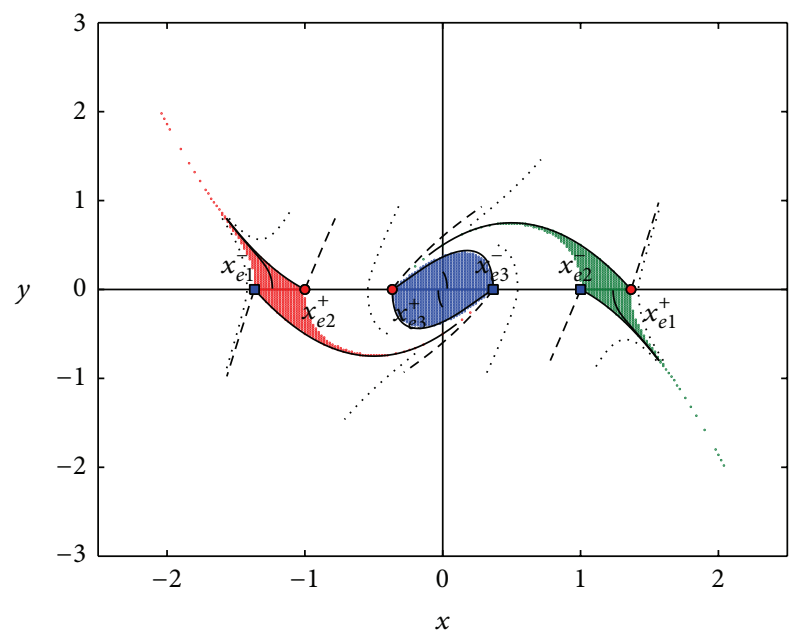

FIGURE 16: The phase portrait of case B1 at $\delta=\delta_{c r_{C}} \approx-0.8660$. Thick - stable separatrix, thick - - - unstable separatrix, thick - $\cdot$ unstable limit cycle, - stable trajectory, $\cdots$ unstable trajectory and coloured dots present the basin of attraction of different attracting sets. Actual parameters' values: $\omega=\sqrt{3} ; \gamma=1 ; \varepsilon=-2 ; \delta=-0.8660 \approx \delta_{{ }_{c r} r_{C}}$.

5.2.2. Subcase B2. One has $\mathscr{D}=0$. The phase portraits, Figure 17, are qualitatively similar till $\delta=\delta_{c r}^{B 2}$ where the trajectory which ends up in equivalent point $x_{e 1}^{+}$starts in $x_{e 2}^{+}$and thus becomes a heteroclinic orbit, Figure 18, instead in the second quadrant of the phase space. The phase space does not change quantitatively with decreasing the value of $\delta$ further on, Figure 19.

5.2.3. Subcase B3. One has $\mathscr{D}>0$. The nature of two equilibrium points is saddle. Hence, the variation of parameter $\delta$

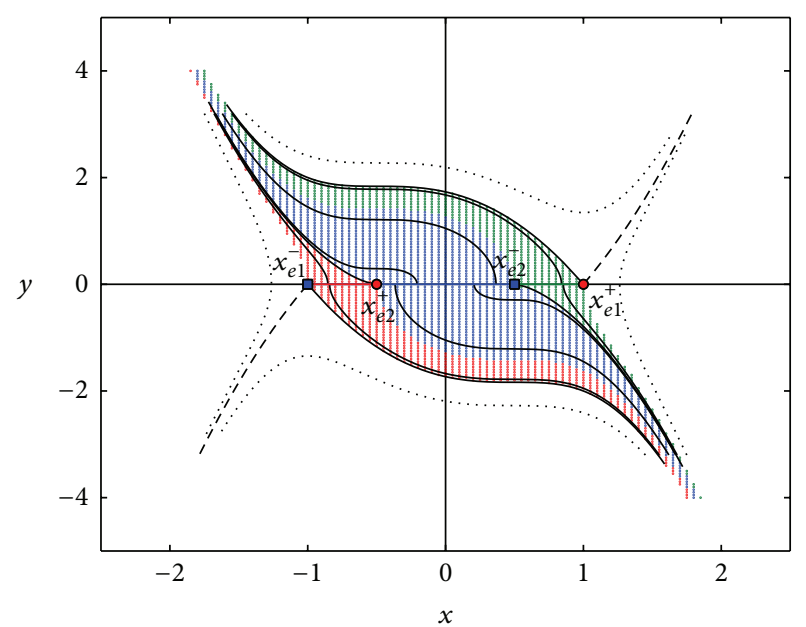

FIgURE 17: The phase portrait of case B2 at $\delta=0$. Thick - stable separatrix, thick - - - unstable separatrix, - stable trajectory, $\cdots$ unstable trajectory and coloured dots present the basin of attraction of different attracting sets. Actual parameters' values: $\omega=\sqrt{3} ; \gamma=1$; $\varepsilon=-4 ; \delta=0$.

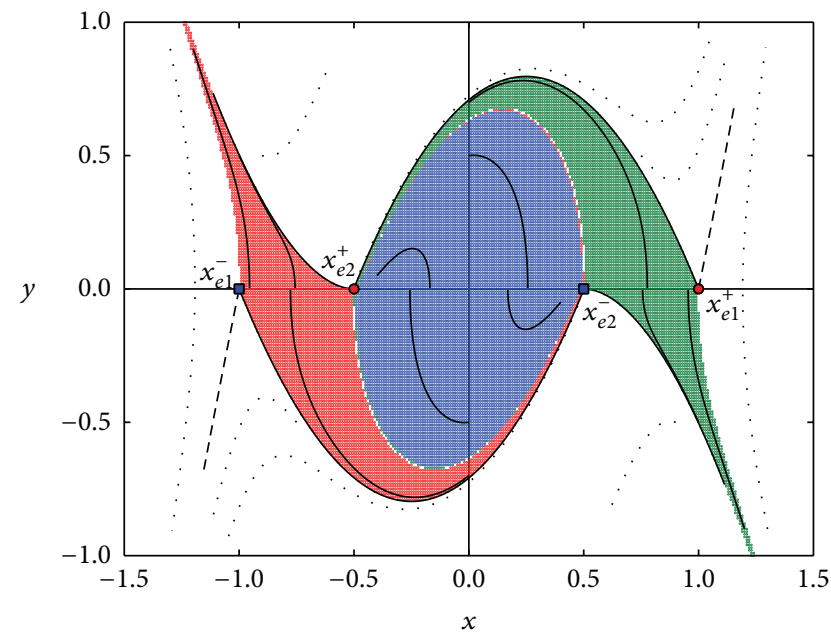

FIgure 18: The phase portrait of case B2 at $\delta=\delta_{c r}^{B 2}$. Thick - stable separatrix, thick - - unstable separatrix, - stable trajectory, $\cdots$ unstable trajectory and coloured dots present the basin of attraction of different attracting sets. Actual parameters' values: $\omega=\sqrt{3} ; \gamma=1$; $\varepsilon=-4 ; \delta=\delta_{c r}^{B 2} \approx-0.611$.

does not change the nature of the points and thus the nature of the phase portrait, Figure 20.

\section{Conclusions}

The paper presents the phase portraits and the sticking regions of the autonomous nonlinear system in its $\delta-\gamma-\varepsilon$ parameter space. The linear system with single-degree-offreedom (SDOF) is upgraded with two widely used nonlinearities: the cubic stiffness $(\varepsilon)$ and the Coulomb dry friction $(\gamma)$ 


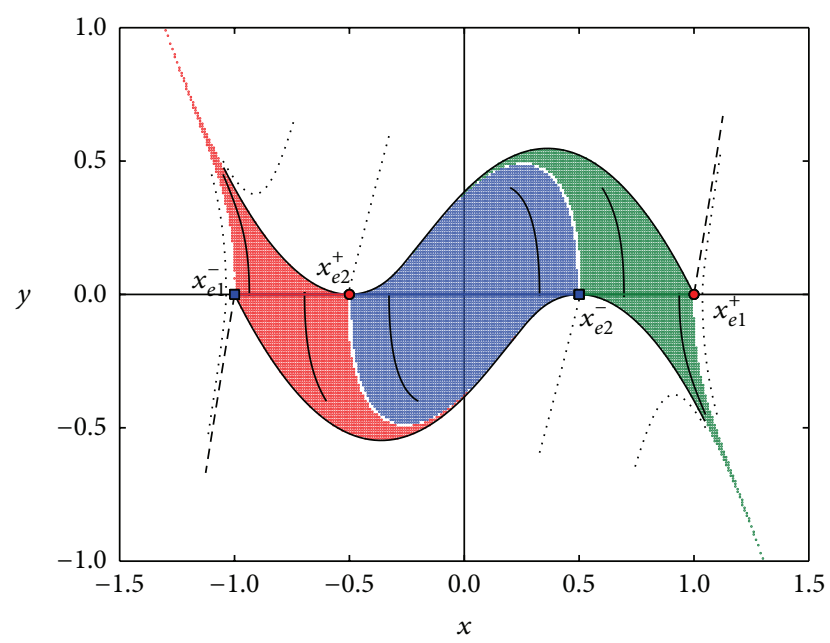

Figure 19: The phase portrait of case B2 at $\delta=-1$. Thick - stable separatrix or heteroclinic orbit, thick - - - unstable separatrix, stable trajectory, $\cdots$ unstable trajectory and coloured dots present the basin of attraction of different attracting sets. Actual parameters' values: $\omega=\sqrt{3} ; \gamma=1 ; \varepsilon=-4 ; \delta=-1$.

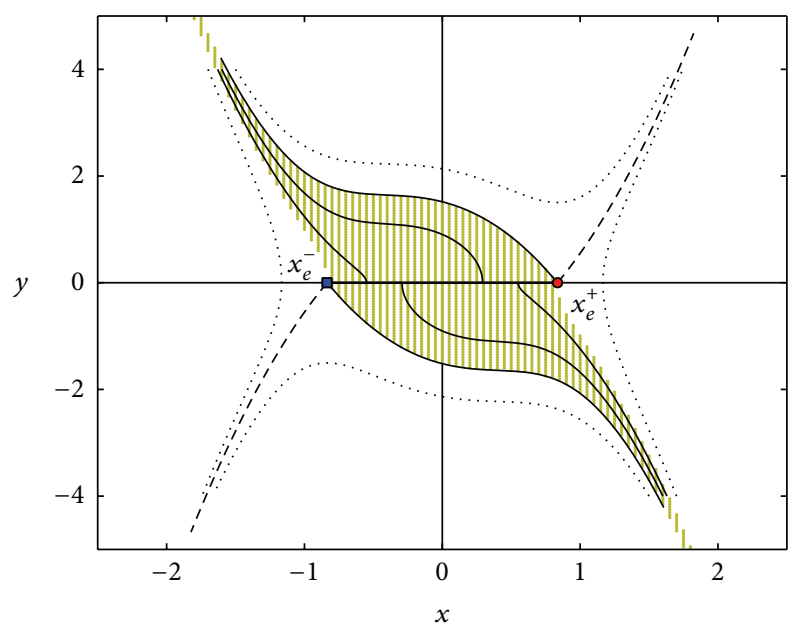

FIGURE 20: The phase portrait of case B3. Thick - stable separatrix, thick - - - unstable separatrix, - stable trajectory, $\cdots$ unstable trajectory and yellow dots present the basin of attraction. Actual parameters' values: $\omega=\sqrt{3} ; \gamma=1 ; \delta=0$.

The phase portrait of the nonlinear system in a way resembles the phase portrait of the Duffing system when $\varepsilon \geq$ 0 . In this case the difference is caused by the Coulomb dry friction model that shifts both phase half-planes in opposite directions.

True difference between Duffing and the system under consideration is shown when $\varepsilon<0$. It was shown that there are three distinctive sticking regions for certain set of parameters and no close basin of attraction for $\delta=0$. When the sticking regions merge together, at the critical combinations of values of the parameters $\gamma$ and $\varepsilon$, an equilibrium point occurs that gives rise to zero eigenvalue of the linearised system.
Even when $\delta<0$ it is evident that, although the equilibrium points, which are at the same time attractors of the system, are unstable, the attracting set(s), which is (are) sticking region(s), still attracts considerable amount of trajectories.

The unstable limit cycle appears in the case of $\delta<0$, in the case of $\varepsilon \geq 0$, and in the case of $\varepsilon<0$ and $\mathscr{D}<0$.

\section{Conflict of Interests}

The author declares that there is no conflict of interests regarding the publication of this paper.

\section{References}

[1] J. M. T. Thompson and H. B. Stewart, Nonlinear Dynamics and Chaos, John Wiley \& Sons, 1991.

[2] N. Jakšić, "Dynamical behavior of a nonlinear single-degreeof-freedom system with negative viscous and positive frictional damping," Meccanica, vol. 43, no. 3, pp. 365-367, 2008.

[3] J. H. G. Macdonald and G. L. Larose, "A unified approach to aerodynamic damping and drag/lift instabilities, and its application to dry inclined cable galloping," Journal of Fluids and Structures, vol. 22, no. 2, pp. 229-252, 2006.

[4] T. Nishihara, S. Kaneko, and T. Watanabe, "Characteristics of fluid dynamic forces acting on a circular cylinder oscillated in the streamwise direction and its wake patterns," Journal of Fluids and Structures, vol. 20, no. 4, pp. 505-518, 2005.

[5] K. Fujita and T. Saito, “Unstable vibration of roller mills," Journal of Sound and Vibration, vol. 297, no. 1-2, pp. 329-350, 2006.

[6] K. V. Avramov and J. Awrejcewicz, "Frictional oscillations under the action of almost periodic excitation," Meccanica, vol. 41, no. 2, pp. 119-142, 2006.

[7] J. P. den Hartog, "Forced vibrations with combined coulomb and viscous friction," Transactions of the ASME, vol. 53, pp. 107$115,1931$.

[8] S. W. Shaw, "On the dynamic response of a system with dry friction," Journal of Sound and Vibration, vol. 108, no. 2, pp. 305$325,1986$.

[9] F. Sorge, "On the frequency behaviour, stability and isolation properties of dry friction oscillators," Meccanica, vol. 42, no. 1, pp. 61-75, 2007.

[10] F. Badrakhan, "Separation and determination of combined dampings from free vibrations," Journal of Sound and Vibration, vol. 100, no. 2, pp. 243-255, 1985.

[11] N. Jakšić and M. Boltežar, "An approach to parameter identification for a single-degree-of-freedom dynamical system based on short free acceleration response," Journal of Sound and Vibration, vol. 250, no. 3, pp. 465-483, 2002.

[12] N. Jakšić, "Power law damping parameter identification," Journal of Sound and Vibration, vol. 330, no. 24, pp. 5878-5893, 2011.

[13] J. P. Baltanas, J. L. Trueba, and M. A. F. Sanjuan, "Power law damping parameter identification," Journal of Sound and Vibration, vol. 330, no. 24, pp. 5878-5893, 2011.

[14] I. Kovacic and M. J. Brennan, The Duffing Equation: Nonlinear Oscillators and their Behaviour, John Wiley \& Sons, 2011.

[15] N. Jakić, Parameter identification of the second-order autonomous dynamical system [Ph.D. thesis], University of Ljubljana, Ljubljana, Slovenia, 2002. 
[16] J. D. Crawford, "Introduction to bifurcation theory," Reviews of Modern Physics, vol. 63, no. 4, pp. 991-1037, 1991.

[17] J. J. Thomsen, Vibrations and Stability: Order and Chaos, McGraw-Hill, 1997.

[18] A. Panfilov, Non-Linear Dynamical Systems, Utrecht University, Utrecht, The Netherlands, 2001. 

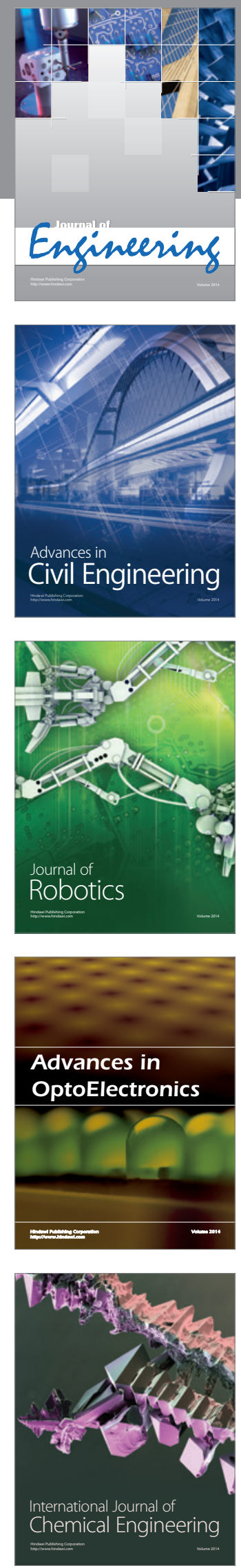

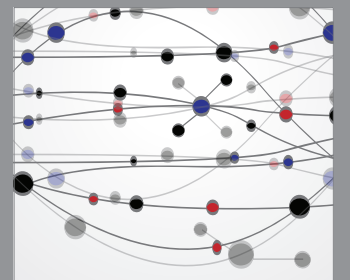

The Scientific World Journal
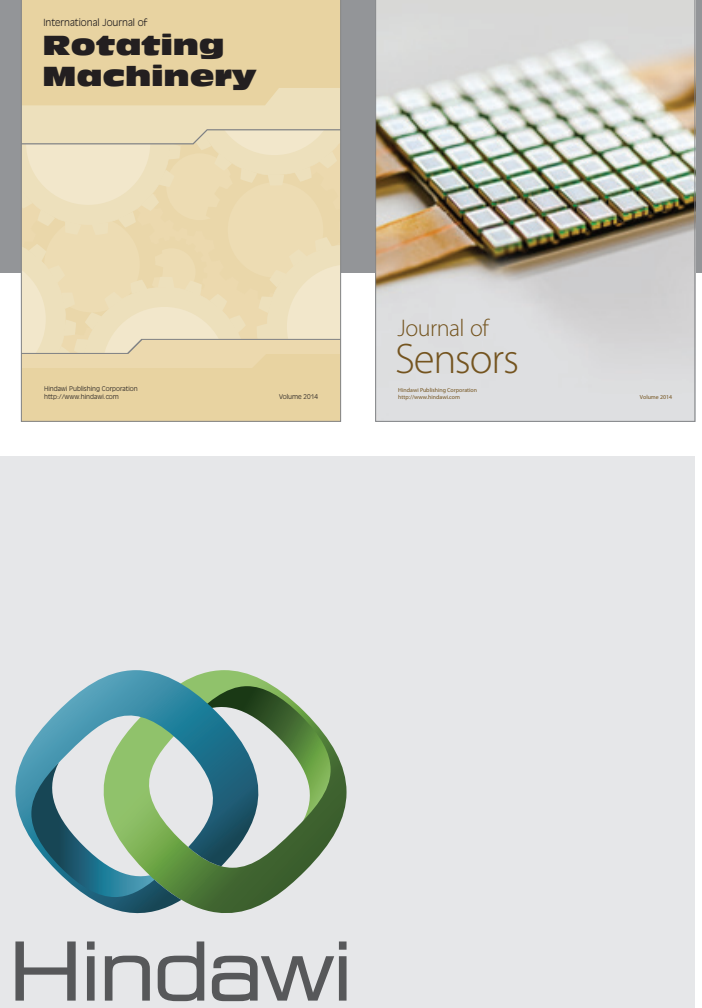

Submit your manuscripts at http://www.hindawi.com
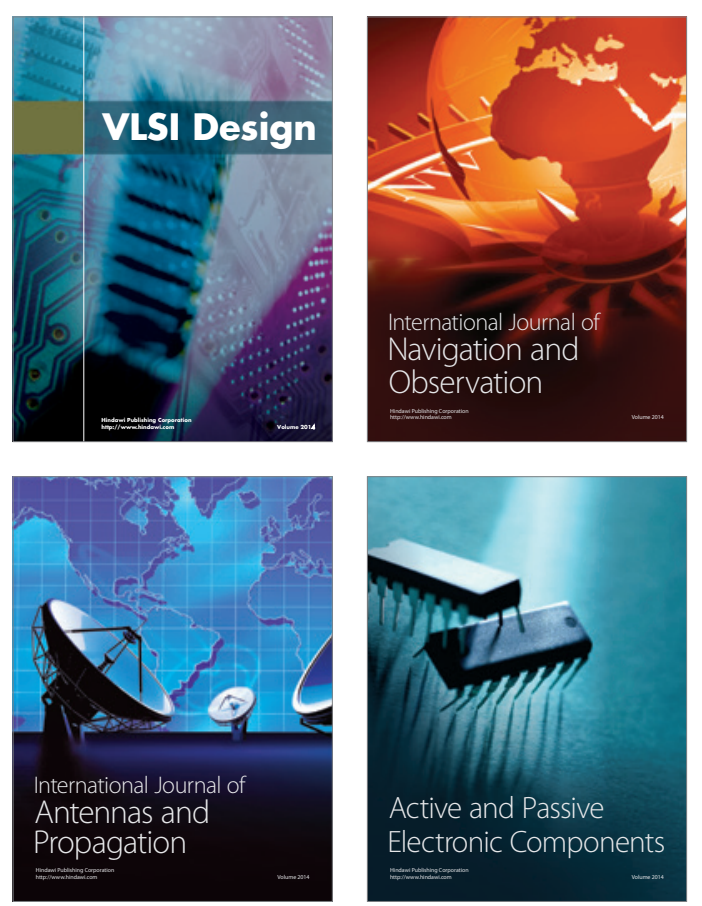
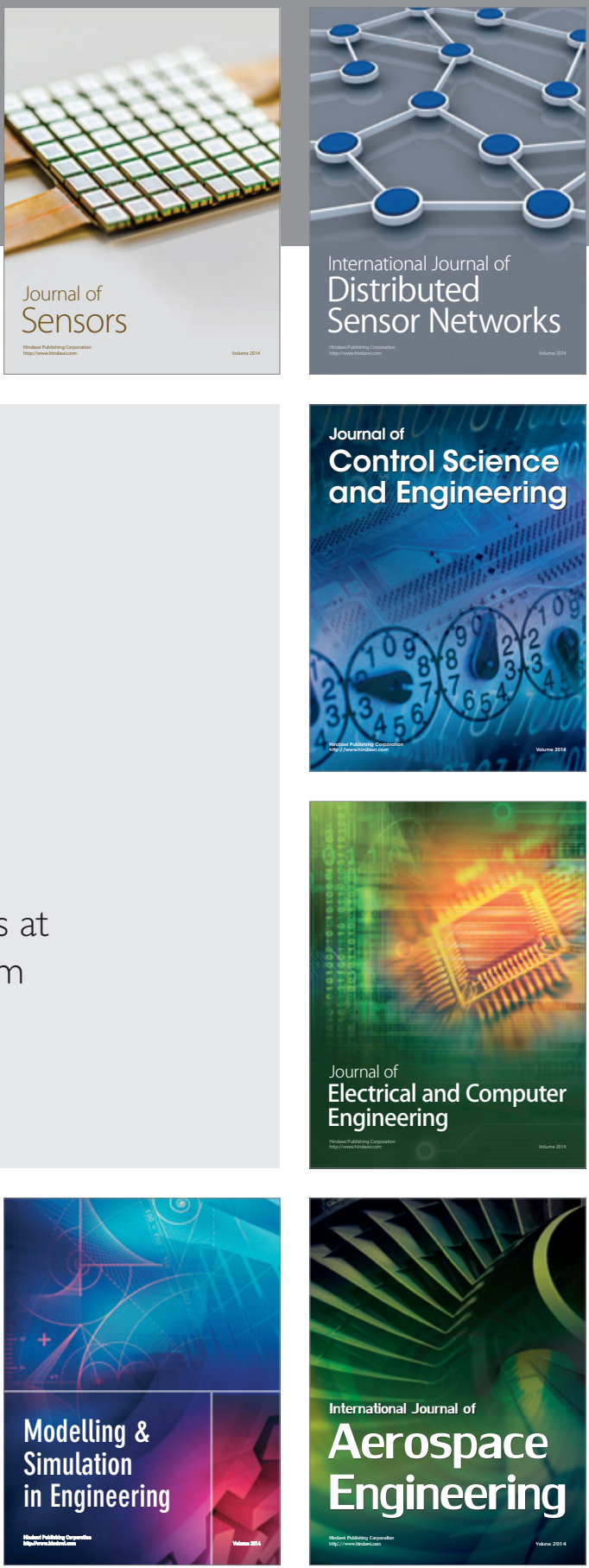

Journal of

Control Science

and Engineering
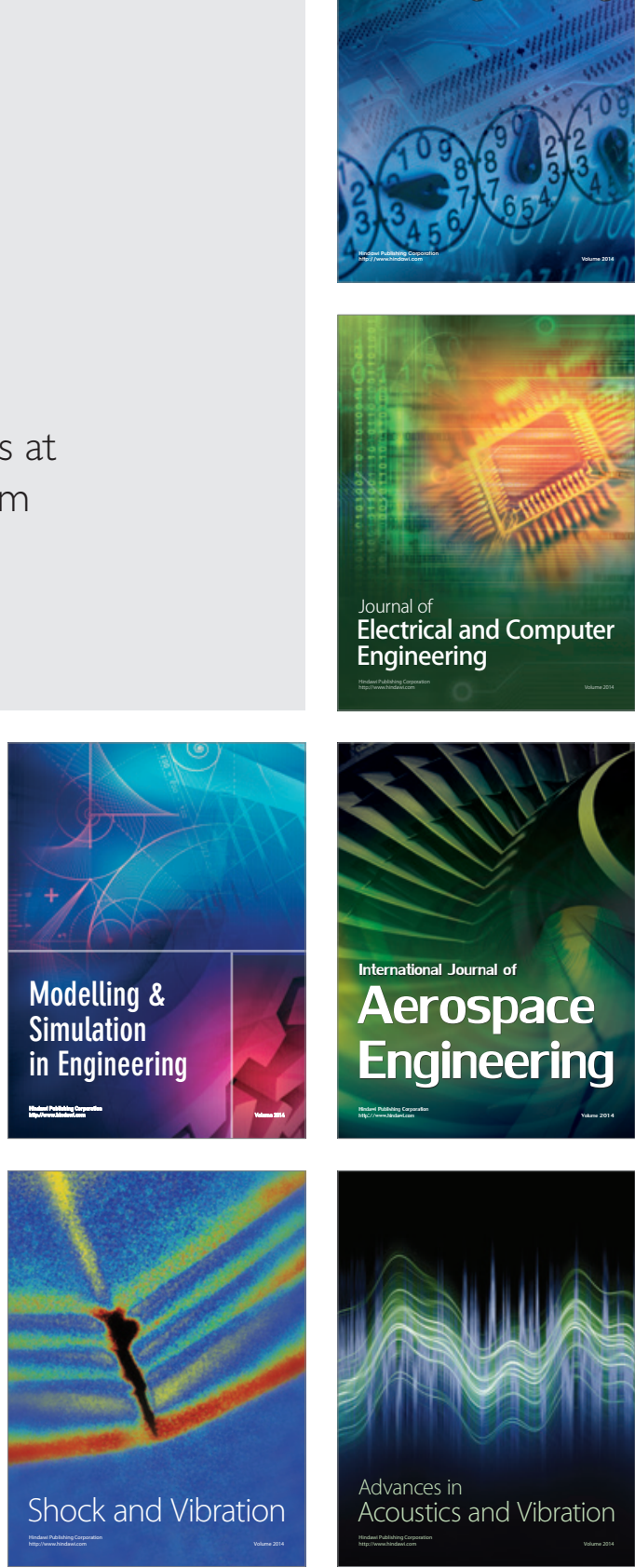